\title{
Research and WebGIS based System Design of Flight Monitoring based on ADS-B
}

\author{
Yubin $X U^{1, a}$, Jie $Y_{A N G}{ }^{1}$, Kun $L^{1}{ }^{1}$, Jing $G^{1} O^{1}$, Yan MA ${ }^{1}$, Yuncheng $\mathrm{HE}^{1}$ \\ ${ }^{1}$ China Academy of Civil Aviation of Science and Technology, Beijing, 100028, China \\ aemail: xuyubin0450@163.com
}

Keywords: ADS-B, WebGIS, Flight Monitoring, GNSS, Message Service

\begin{abstract}
This article aims to give general description of principle, application and problems of ADS-B technology, which is mainly developed and used for civil aviation. Based on the advantages of ADS-B, more and more applications are being derived, especially in aircraft surveillance. There are some world known products like Flightradar24, Flightaware, PlaneFinder, and etc. However, these systems cover wide area of the world, China not included. Because there is few ground stations in China, and ground station is not permitted to be constructed by private companies. In this article, ground stations deployment, network, data communication are mainly discussed. Meanwhile, we developed relevant applications, respectively flight monitoring system. System framework, functions and technique details were described. Future application and prospects of ADS-B in civil aviation were debated at last.
\end{abstract}

\section{Introduction}

Automatic dependent surveillance (ADS) is defined by ICAO (International Civil Aviation Organization) as, "A surveillance technique in which aircraft automatically provide, via a data link, data derived from on-board navigation and position-fixing systems, including aircraft identification, four dimensional position, and additional data as appropriate." In ADS, the aircraft position calculation is finished on board, not on the ground data center [1].

Automatic dependent surveillance-broadcast (ADS-B)[2] is developed from Europe and America. It is an important communication protocol of modern civil aviation. And it will be indispensable part of next generation air transportation system. With more and more aircrafts in airspace, the horizontal separation and vertical separation is being smaller and smaller. Surveillance methods are playing essential roles in air traffic management (ATM) under these circumstances. According to statistics of Alaska Airline [3], the accidents have been reduced by about 60\%. Route control separation has been greatly reduced from 80 nautical miles to 5 nautical miles, which helped to increase aircrafts density and airspace capacity.

In order to use and reveal ADS-B data very well, WebGIS will be adopted to develop relevant software, which is going to meet different demands of users. Based on WebGIS, users can: a) can assess the service where they have internet There is no limitation of location. b) Users can assess Web GIS system once they possess universal web browsers, no matter what kinds of machines server and clients are. c) Unlike traditional GIS, Web GIS reduce the cost of professional software enormously.

In the article, the structure of ground monitoring station deployment was described. Through the network of ground stations, the coverage of surveillance will be expanded. All data will reach national data processing center finally. The framework of software is divided into several parts, or sensor, ground monitoring station, local data server, communication network, secondary data processing center and national data processing center. And all request can be handled by WebGIS platform.

\section{ADS-B Principle and Applications}

As one of main surveillance method, ADS-B belongs to cooperative dependent surveillance [4]. 
And automatic dependent surveillance contract (ADS-C) is also the member of this category. There are four specific features for ADS-B, respectively automatic with no pilot action, dependent on Global Navigation Satellite System (GNSS), surveillance with good position accuracy and multiple information, and broadcasting to any equipment possessing the ability to receive.

Table 1 gives the comparison between two members of ADS family. From the table, we can get that ADS-B is a very cheap surveillance method. And ADS-C is mainly used during the oceanic route or some routes there is few ground stations. On the contrast, ADS-C is of great cost and low update period.

Table 1 the comparison between ADS-B and ADS-C

\begin{tabular}{cll}
\hline & ADS-B & ADS-C \\
\hline $\begin{array}{c}\text { Transmission } \\
\text { model }\end{array}$ & Point to point & Broadcast \\
\hline Acknowledgement & Yes & No \\
\hline Data Link & $\begin{array}{l}\text { VDL1 , VDL2 } \\
\text { SATCOM,HFDL,VDL4 }\end{array}$ & 1090ES,UAT, VDL4 \\
\hline Message Rate & 64s to several minutes & 0.5s \\
\hline $\begin{array}{c}\text { air-ground com } \\
\text { cost }\end{array}$ & $\begin{array}{l}\text { SITA or ARINC } \\
\text { By airlines }\end{array}$ & No \\
\hline $\begin{array}{c}\text { Ground users } \\
\text { limits }\end{array}$ & 5 max & No \\
\hline $\begin{array}{c}\text { Coverage } \\
\text { Air-air application }\end{array}$ & No & Continental (range \\
\hline Related service & CPDLC & 200NM) \\
\hline
\end{tabular}

\section{A. Principle}

The following figure describes basic principle of ADS-B. ADS-B can share some parts with secondary radar transponder, especially the download data link (both same as 1090ES). In most of time, the functional modules are integrated into the $\mathrm{S}$ mode transponder, which could also be an independent equipment for aircrafts. The principle is very simple, GNSS signals will be received by GNSS antenna which is with transponder firstly. Then three dimensional position of aircraft will be calculated by positioning modules. After that, the position will be packaged with other information like aircraft address, flight status, altitude, etc. The zipped message will be broadcasted through data link 1090ES.

From the direction of message transmission, ADS-B is split into ADS-B IN and ADS-B OUT. There are three main data link for ADS-B data transmission, or extender squitter (1090ES), VDL mode 4 and UAT. 1090ES is recognized as the worldwide ADS-B data-link media by ICAO, Eurocontrol, FAA and Australia. It's also the only authorized data link in China. VDL mode 4 is just supported by North European countries and Russia (for light aviation). And UAT data link is only chosen by FAA for general aviation.

\section{B. Applications}

(1) Air Traffic Control

ADS-B is going to be used for air traffic management via broadcasting information like ID, position, altitude, and other message periodically. Controllers can have a whole control of flight status of different aircrafts [5]. ADS-B is going to make controllers' work simpler and has reduced the cost greatly.

(2) Surface surveillance and collision avoidance

With more and more aircrafts on the surface, the surveillance is mandatory. ADS-B is such a way with low cost and high data update rate. Based on DGPS (Differential GPS), we can obtain position with higher accuracy for aircrafts. The combination with other technologies like real time video monitoring will provide firm assurance for the surface safety.

(3) Aircraft conflicts detection 
During one flight's operation, it's very important to detect the conflicts with other aircrafts or vehicles efficiently. As ADS-B possesses high accuracy of spatial position, real time ADS-B data can be utilized to detect these conflicts. Conflict will be alerted and handled once it is detected [4][6].

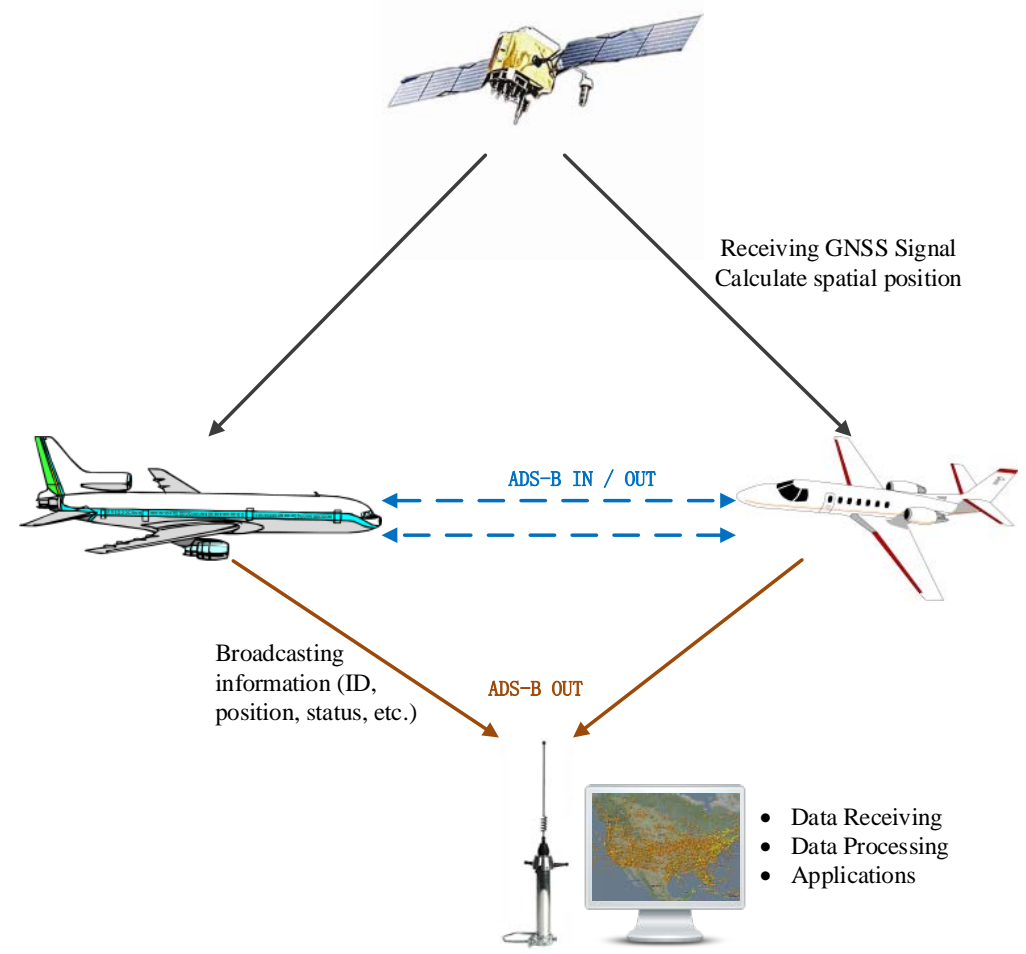

Fig. 1. Basic principle of ADS-B technology

\section{Ground Stations Deployment Framework}

\section{A. Framework of ground station deployment}

Ground station is the cornerstone for ADS-B applications. ADS-B signal will reach ground station via antenna fixed in aircrafts. Figure 2 describes the physical structure for construction of ground station and data processing center. From the graph, ground monitoring station is closely bound with local server. There are three categories for data processing center.

Monitoring station: it is designed to monitor both transport aircrafts and general aircrafts through decoding ADS-B message from aircrafts. The maximum distance which ground station can receive is about $350 \sim 400 \mathrm{~km}$.

(1) Local data server

This is also called the basic data processing center, which is always a normal server. Received signal will be decoded in this server. And ADS-B data can be stored into database under certain time span if in need.

(2) Secondary data processing center

This kind of data processing center will store and handle data from 10 local data server. The deployment of secondary data processing center is to confirm the data availability and share the responsibility of primary data center. Meanwhile, it can reduce the burden of primary data center.

(3) Primary data processing center

All data will aggregate into the center, which is also national data processing center essentially. It possesses the most complete surveillance data under the coverage of ground station networks. All data request can be handled with this center. 


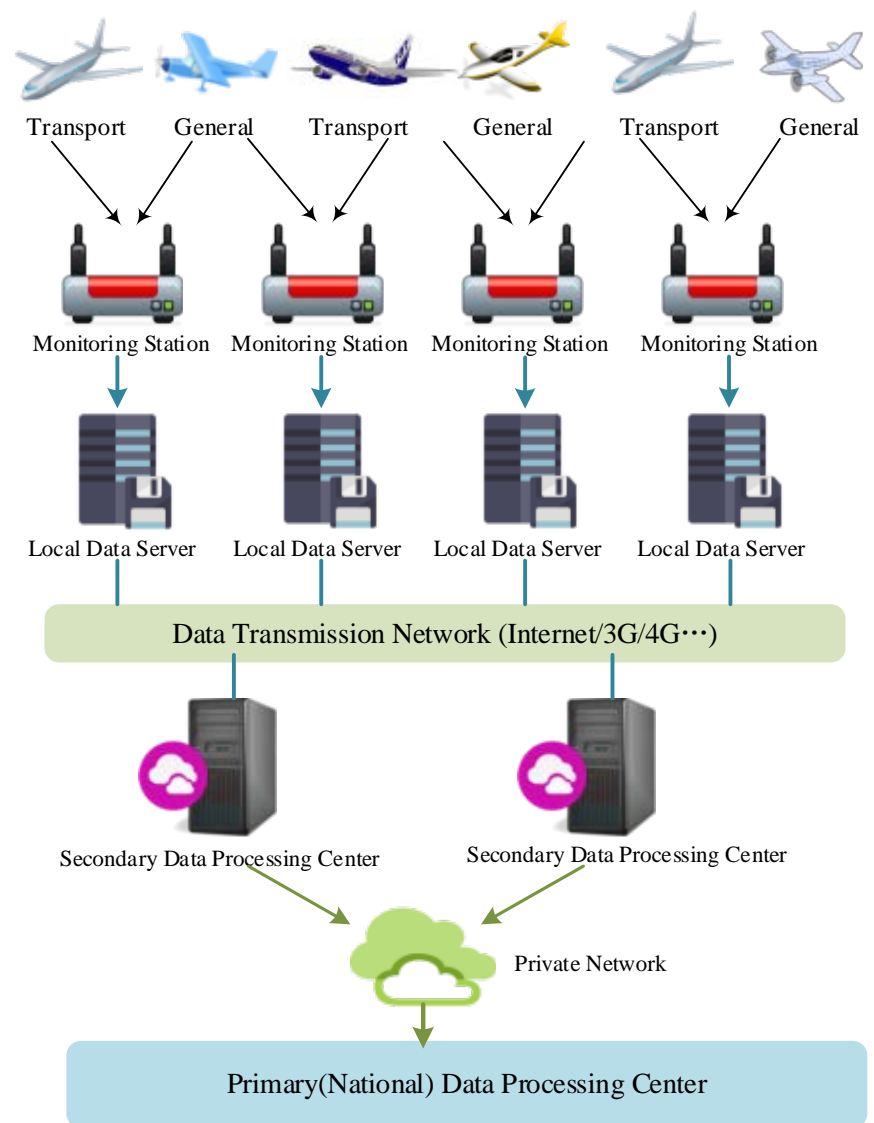

Fig. 2. Network structure of ground monitoring stations

\section{B. Ground station}

ADS-B air-ground data exchange is going to be finished by aircrafts and ground stations. Data link is 1090ES specified by ICAO.

In figure 3, (a) shows the appearance of antenna. And (b) is the outside view of ground monitoring station, which is of standard size for use as server. The main features of ADS-B ground stations are listed as followings: 1) Double receiving channel to ensure high availability. 2) Operation and maintenance can be done from remote computer. There is no need for worker on the station except hardware breakdown. 3) Different ground stations could be put into a network to enhance surveillance. 4) The embedded software structure is flexible, revealing ADS-B information from various perspectives.

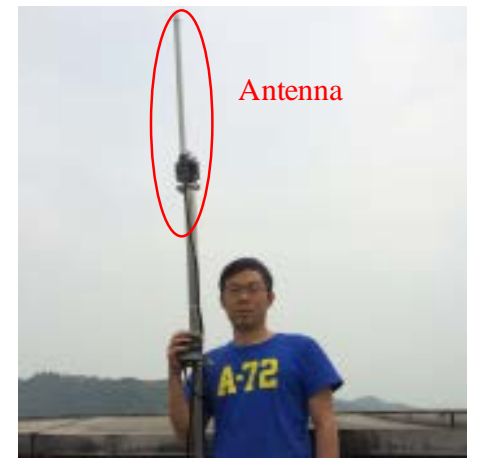

(a) ADS-B ground antenna

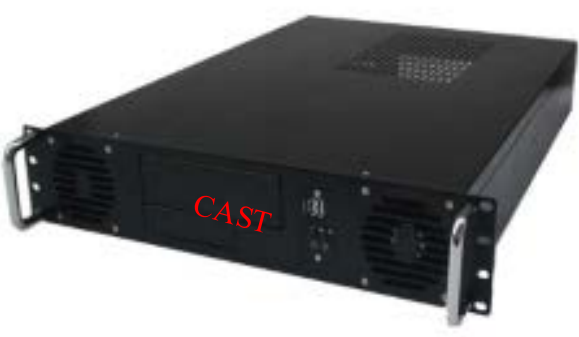

(b) Ground monitoring station server

Fig. 3. ADS-B ground antenna and monitoring station server 


\section{Flight Monitoring System Design and Implementation}

\section{A. Framework of flight monitoring system}

The framework is consisted of four layers, respectively database layer, WebGIS platform, network layer and users.

(1) Database layer

All ADS-B data from ground stations will be stored into the database. There will be about 2 4GB data to be transferred to data server in China Academy of Civil Aviation Science and Technology (CAST) each day. To guarantee the integrity and accidental use of data, backup data storage is also approached. Even the main database server is broke down, the system could be in work seamlessly. All applications will be developed on the basis of ADS-B data received.

(2) WebGIS platform

The platform is aimed to handle the request from web clients or mobile clients. Real time ADS-B data under specific filters will be distributed to the relevant client to monitor interested flights. Clients can maintain flights id, position, speed, altitude of certain aircraft from WebGIS platform. Meanwhile, you can apply for the historic track or residual fuel of the flights you're interested in.

(3) Network

Network layer is indispensable for WebGIS applications. Because you need to visit relevant data from web browsers in your computer. You need to use APP in your pads or phones via network to maintain data. In network layer, Internet and wireless network (Wi-Fi / 2G / 3G /4G) are both included.

(4) Users

The service will be provided to different users. There are four categories for users, or airport, air traffic controllers, airline companies, and public users.

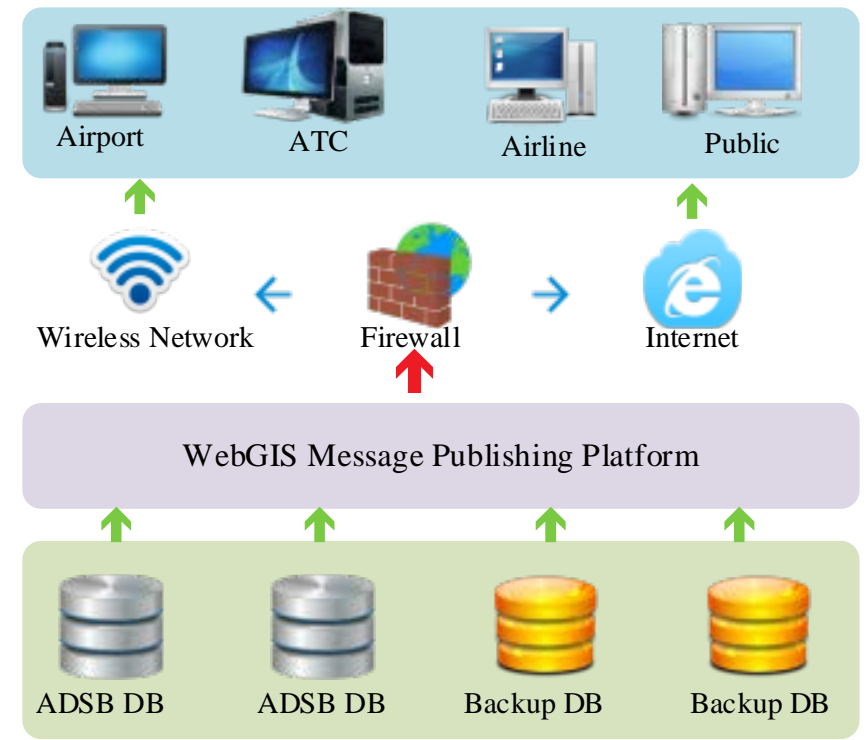

Fig. 4. Framework of ADS-B software

\section{B. System function design}

(1) Geographic data management

The basic geographic data are consisted of administration map, satellite map, geographic terrain map and airport map. Then for different users, different kinds of thematic data will be provided. Thematic data comprises departure procedure map, approach procedure map, route map, air traffic control map, and so on.

Users can customize their own geographic map through selecting data they are interested. All geographic data layer can be seen from data management panel. 
(2) Flight monitoring

All the aircrafts with ADS-B modules will be detected when the instrument in on, the preconditions is that the aircrafts are under the coverage of certain ground monitor station. The spatial position will be marked on the map. Relevant information like speed, altitude, flight status can be checked. This make it visible on computer and improve management efficiency.

(3) Historic track management

All ADS-B data will be stored into database. For the reason of big size for each day's data, data three months ago will be cleared from hard disks. Historic track can be requested from WebGIS platform based on the historic data.

(4) Flight landing time

Based on historic flight data, the flight landing time will be estimated from about $50 \mathrm{~km}$ away from the airport where the flight is going to land. Through the combination of multiple flights' landing records, the error would be controlled as less as 30 seconds under normal circumstances. The exception contains going around, not following the approach procedure, etc.

\section{System implementation}

Based on the framework of software and data from ground station, WFMS (web-GIS based flight monitoring system) was developed. The application is accessible from web explorer, which means users can visit data and check aircraft position anywhere there is network. Figure 5 and 6 reveal the user interface.

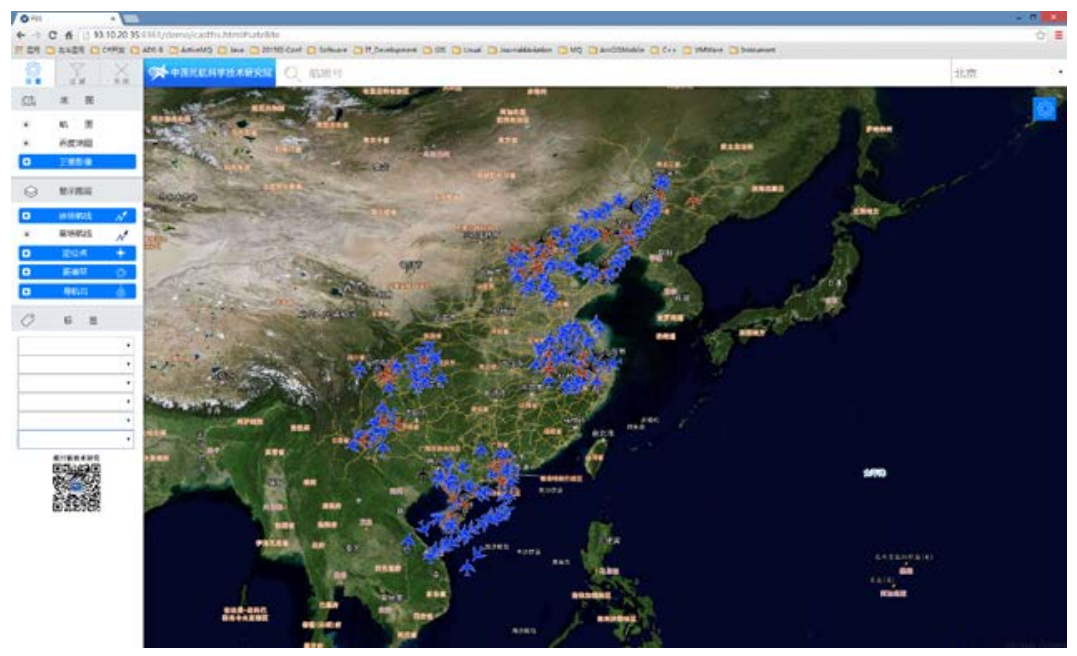

Fig. 5. User interface of the ADS-B system

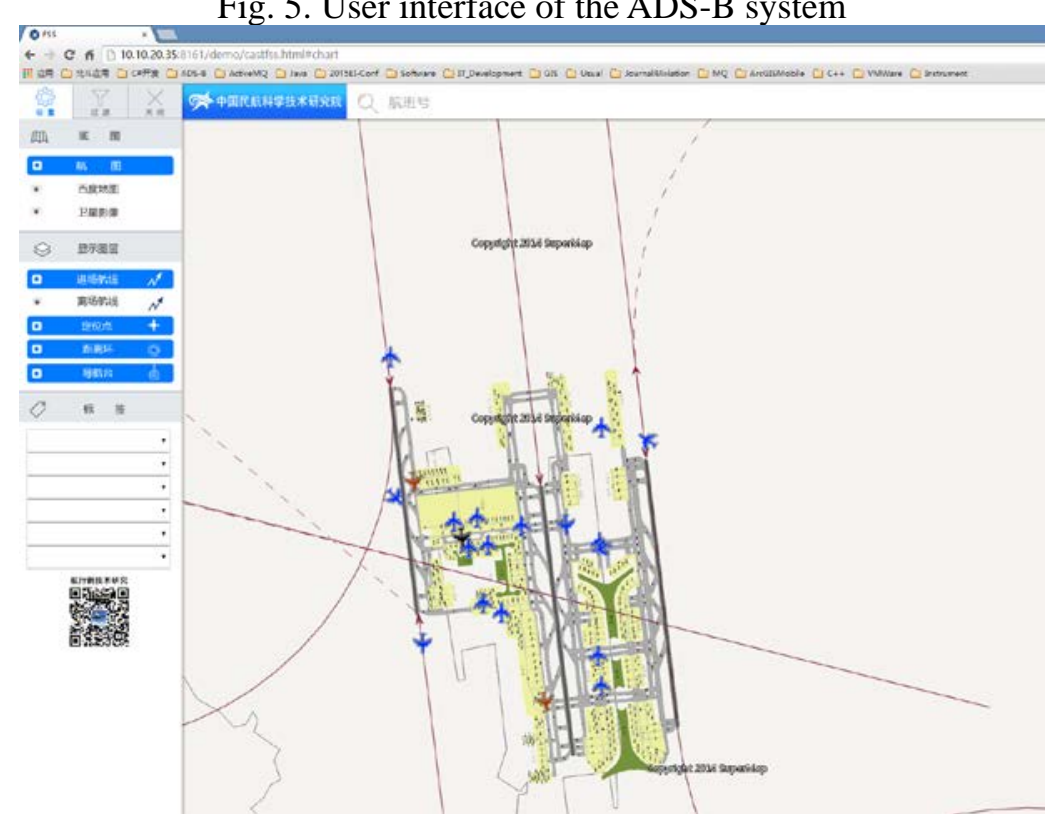

Fig. 6. Airport element in the ADS-B system 


\section{Conclusion}

ADS-B is a high efficient method to approach aircrafts surveillance. Especially in non-radar areas, it will be the unique way to catch where aircrafts are. It will grant guidance for terminal approach and departure where is not suitable for installing radar facilities. Compared with radar surveillance, ADS-B possesses higher data update rate. Position message is updated twice in one second. It will be a supplement and backup for surveillance in radar areas. It will also be of great help for surface monitoring, alerting when runway is invaded or conflict between aircrafts and airport vehicle is detected. Therefore, ADSB can enhanced air traffic security greatly [7-8].

Based the advantages of ADS-B, lots of work have been done by CAST, including ground stations deployment and software development. For the time being, 33 ground stations have been installed in metropolitan airports. Two secondary data processing center and national data processing center have been constructed. Mobile flight monitoring system has been developed and is in test phase at present. ADS-B technology is proved to be a high-availability and efficient way to approach surveillance.

In future, there will a plenty of applications derived from ADS-B data. For example, pilot behavior can be studied on the basis of giant ADS-B data. Residual fuel can be calculated precisely from multiple relevant parameters of flights. The behavior of crossing boundary can be caught. And the ADS-B data will also provide great help for incident investigations.

However, there is some deficiencies for ADS-B technology. The latency can't be avoided. Original data has to be through several transmission process, which increase the delay. The usual delay is about 1.5 seconds. Another reason that you can't correct the delay is that there is no time stamp for ADS-B message, which is the bottleneck for ADS-B application. Lots of work will be approached on this aspect.

\section{Acknowledgements}

The research work was supported by National Natural Science Foundation of China under Grant No. U1333203 and U1333124.

\section{Reference}

[1] FAA, Concept of operations for the next generation air transportation system. JPDO, 2007. Version 2.0.

[2] FAA, Automatic Dependent Surveillance-Broadcast (ADS-B) Out Performance Requirements to Support Air Traffic Control (ATC) Service: Final Rule. 14 CFR Part 91, 2010. Federal Register 75(103).

[3] Li, W. and P. Kamal. Integrated aviation security for defense-in-depth of next generation air transportation system. Technologies for Homeland Security (HST), 2011 IEEE International Conference on. 2011.

[4] Orefice, M., et al. Aircraft conflict detection based on ADS-B surveillance data. in Metrology for Aerospace (Metro Aero Space), 2014 IEEE. 2014. IEEE.

[5] Finke, C., et al., Enhancing the security of aircraft surveillance in the next generation air traffic control system. International Journal of Critical Infrastructure Protection, 2013. 6(1): p. 3-11.

[6] Gariel, M., F. Kunzi, and R.J. Hansman. An algorithm for conflict detection in dense traffic using ADS-B. in Digital Avionics Systems Conference (DASC), 2011 IEEE/AIAA 30th. 2011.

[7] McCallie, D., J. Butts, and R. Mills, Security analysis of the ADS-B implementation in the next generation air transportation system. International Journal of Critical Infrastructure Protection, 2011. 4(2): p. $78-87$ 
[8] Lu, Y., C. Liu, and P. Liu. An improved tracking method based on data mining in ADS-B for surface surveillance. in Fuzzy Systems and Knowledge Discovery (FSKD), 2012 9th International Conference on. 2012. IEEE. 